\title{
Deep Brain Stimulation of the Center Median-Parafascicular Complex of the Thalamus Has Efficient Anti-Parkinsonian Action Associated with Widespread Cellular Responses in the Basal Ganglia Network in a Rat Model of Parkinson's Disease
}

\author{
Loréline Jouve, Pascal Salin, Christophe Melon, and Lydia Kerkerian-Le Goff \\ Developmental Biology Institute of Marseille Luminy, Unité Mixte de Recherche 6216 Centre National de la Recherche Scientifique-Université de la \\ Méditerranée, 13288 Marseille, France
}

\begin{abstract}
The thalamic centromedian-parafascicular (CM/Pf) complex, mainly represented by $\mathrm{Pf}$ in rodents, is proposed as an interesting target for the neurosurgical treatment of movement disorders, including Parkinson's disease. In this study, we examined the functional impact of subchronic high-frequency stimulation (HFS) of Pf in the 6-hydroxydopamine-lesioned hemiparkinsonian rat model. Pf-HFS had significant anti-akinetic action, evidenced by alleviation of limb use asymmetry (cylinder test). Whereas this anti-akinetic action was moderate, Pf-HFS totally reversed lateralized neglect (corridor task), suggesting potent action on sensorimotor integration. At the cellular level, Pf-HFS partially reversed the dopamine denervation-induced increase in striatal preproenkephalin A mRNA levels, a marker of the neurons of the indirect pathway, without interfering with the markers of the direct pathway (preprotachykinin and preprodynorphin). Pf-HFS totally reversed the lesion-induced changes in the gene expression of cytochrome oxidase subunit I in the subthalamic nucleus, the globus pallidus, and the substantia nigra pars reticulata, and partially in the entopeduncular nucleus. Unlike HFS of the subthalamic nucleus, Pf-HFS did not induce per se dyskinesias and directly, although partially, alleviated L-3,4dihydroxyphenylalanine (L-DOPA)-induced forelimb dyskinesia. Conversely, L-DOPA treatment negatively interfered with the antiparkinsonian effect of Pf-HFS. Altogether, these data show that Pf-DBS, by recruiting a large basal ganglia circuitry, provides moderate to strong anti-parkinsonian benefits that might, however, be affected by L-DOPA. The widespread behavioral and cellular outcomes of Pf-HFS evidenced here demonstrate that CM/Pf is an important node for modulating the pathophysiological functioning of basal ganglia and related disorders.
\end{abstract}

\section{Introduction}

The center median-parafascicular complex (CM/Pf) of the thalamus, mainly represented by the Pf in rodents, differs from the other intralaminar thalamic groups in its rich connectivity with the basal ganglia (BG). It is the source of a major excitatory glutamatergic input to the striatum (Smith et al., 2004, 2009), which modulates large striatal neuronal populations through local processing (Nanda et al., 2009). CM/Pf also provides projections to all the other BG components (Van der Werf et al., 2002) and, in turn, receives substantial innervation from the BG output structures (Sidibé et al., 2002), particularly from the internal globus

Received March 18, 2010; revised May 4, 2010; accepted June 11, 2010.

This work was supported by grants from the Centre National de la Recherche Scientifique, Université de la Méditerranée, Fédération pour la Recherche sur le Cerveau and France Parkinson. L.J. was supported by a grant from the French Ministry of Education and Research.

Correspondence should be addressed to Lydia Kerkerian-Le Goff, Team "Cellular Interactions, Neurodegeneration and Neuroplasticity," Developmental Biology Institute of Marseille-Luminy, UMR 6216 CNRS-Université de la Méditerranée, Case 907, Parc scientifique de Luminy, 13288 Marseille Cedex 9, France. E-mail: kerkerian@ibdml.univ-mrs.fr.

DOl:10.1523/JNEUROSCI.1404-10.2010

Copyright $\odot 2010$ the authors $\quad$ 0270-6474/10/309919-10\$15.00/0 pallidus (GPi), called the entopeduncular nucleus (EP) in rodents. One of the main functions attributed to the $\mathrm{CM} / \mathrm{Pf}-\mathrm{BG}$ circuit is the process of attentional orienting and action selection appropriate for unexpected situations (Raeva, 2006; Minamimoto et al., 2009).

Long "forgotten" in the schemes of BG anatomo-functional organization, $\mathrm{CM} / \mathrm{Pf}$ has recently been attracting increasing interest in the context of BG-related functions and disorders. In particular, there is accumulating evidence for $\mathrm{CM} / \mathrm{Pf}$ involvement in Parkinson's disease (PD). Important neurodegeneration in $\mathrm{CM} / \mathrm{Pf}$ has been reported in PD patients (Henderson et al., 2000). In rodent PD models, neurodegeneration and complex alterations of $\mathrm{CM} / \mathrm{Pf}$ activity have been evidenced (Freyaldenhoven et al., 1997; Orieux et al., 2000; Aymerich et al., 2006; ParrBrownlie et al., 2009; Sedaghat et al., 2009). Because extensive Pf lesion has been shown to prevent most of the cellular alterations associated with dopamine depletion in the rat $\mathrm{BG}$ network, reactive changes in the activity of spared CM/Pf neurons rather than $\mathrm{CM} / \mathrm{Pf}$ neurodegeneration might contribute to the pathophysiological functioning of BG in the PD state (Bacci et al., 2004). This 
hypothesis complies with casual clinical reports that had designed CM/Pf as a candidate target for the neurosurgical treatment of movement disorders (Caparros-Lefebvre et al., 1999; Krauss et al., 2002). To date, we are far from a comprehensive view of the therapeutic potential of CM/Pf surgery. Whereas $\mathrm{CM} / \mathrm{Pf}$ lesion in the MPTP monkey model of PD provided no stable benefit on the parkinsonian syndrome (Lanciego et al., 2008), recent trials of multitarget deep brain stimulation (DBS) in small cohorts of PD patients have described some specific CM/Pf-mediated effects compared with current targets with excellent anti-akinetic or anti-dyskinetic action: subthalamic nucleus (STN) and GPi, respectively. Efficient, although much less potent on unified Parkinson's disease rating scale (UPDRS) scores and L-3,4-dihydroxyphenylalanine (LDOPA)-induced dyskinesias (LIDs), CM/Pf-DBS at high frequency [i.e., high-frequency stimulation (HFS)] strongly relieves tremor and is efficient on freezing (Mazzone et al., 2006; Pepe et al., 2008; Stefani et al., 2009).

Here, we investigated the functional outcomes of subchronic Pf-HFS in comparison with previous or parallel data obtained for STN-HFS in the 6-hydroxydopamine-lesioned hemiparkinsonian rat model. We confirm that Pf-HFS relieves akinesia less potently than STN but has direct anti-dyskinetic action. We further found that Pf-HFS has a strong beneficial effect on sensorimotor neglect and that L-DOPA might affect its antiparkinsonian action. Finally, we show that the impact of PfHFS on BG pathophysiological functioning differs mainly from the one of STN-HFS by its action on EP, pointing to the CM/Pf-EP connections as a key substrate for specific CM/Pf-mediated action on movement disorders.

\section{Materials and Methods}

All experiments have been performed on male Wistar rats weighing 160 $180 \mathrm{~g}$ at the time of the first surgery, and were conducted in accordance with the European Communities Council Directive of November 24, 1986 (86/609/EEC). Naive rats without any surgery or treatment served as controls, and animals with the dopamine lesion were divided into five groups: (1) lesion alone without any further treatment (6-OHDA); (2) Pf-HFS for $6 \mathrm{~d}$; (3) Pf-DBS at low frequency [i.e., low-frequency stimulation (LFS; $25 \mathrm{~Hz}$ )] for $6 \mathrm{~d}$; (4) L-DOPA + Pf-HFS (HFS during the last $6 \mathrm{~d}$ of the $21 \mathrm{~d}$ L-DOPA treatment); and (5) STN-HFS for $6 \mathrm{~d}$. All animals were killed 30-37 d after the 6-OHDA lesion. Animals to be included in the analyses were selected a posteriori based on controls of the dopamine denervation extent and correct location of the stimulating electrode. The numbers of animals per group were as follows: 8 controls, 10 6-OHDA, 10 Pf-HFS, 4 Pf-LFS, 8 STN-HFS, and 5 Pf-HFS + L-DOPA.

\section{6-OHDA lesion}

Surgery was performed under Equitesin anesthesia ( $4 \mathrm{ml} / \mathrm{kg})$. Animals received a unilateral injection of $12 \mu \mathrm{g}$ of 6-OHDA (Sigma-Aldrich) dissolved in $6 \mu \mathrm{l}$ of $0.9 \%$ sterile $\mathrm{NaCl}$ containing $0.1 \%$ ascorbic acid, at the rate of $1 \mu \mathrm{l} / \mathrm{min}$, in the left substantia nigra pars compacta. The stereotaxic coordinates of the injection site were as follows: anteroposterior $+2.2 \mathrm{~mm}$, lateral $2.0 \mathrm{~mm}$, dorsoventral $+3.3 \mathrm{~mm}$, with the incisor bar at $+5.0 \mathrm{~mm}$ above the interaural plane, according to the rat stereotaxic atlas by De Groot (1959).

\section{Electrode implantation and chronic DBS}

Fifteen days after the 6-OHDA lesion, the rats to be treated by DBS were unilaterally implanted with one bipolar electrode in the ipsilateral Pf or STN. The material and procedure for the stimulation were the same as previously used for STN-HFS (Oueslati et al., 2007). The stimulating electrode was formed by two parallel platinum iridium wires insulated with Teflon and bared at the extremity on a length of $500 \mu \mathrm{m}$ (diameter of each wire; insulated, $140 \mu \mathrm{m}$; bare, $76 \mu \mathrm{m}$ ). The distance between the two wires was $\sim 400 \mu \mathrm{m}$. The electrode was implanted so that the two wires were placed in the anteroposterior axis and the bared part of each wire was covering major part of the structure of interest in depth. The stereotaxic coordinates were defined from the stereotaxic atlas of Paxinos and Watson (1986) using the following interaural coordinates: anteroposterior $+3.75 \mathrm{~mm}$ (taken at equidistance of the two wires), lateral 1.0 $\mathrm{mm}$, dorsoventral $+4.1 \mathrm{~mm}$, incisor bar at $-3.3 \mathrm{~mm}$ for Pf and anteroposterior $+5.2 \mathrm{~mm}$ (taken at equidistance of the two wires), lateral 2.3 $\mathrm{mm}$, and dorsoventral $+2.4 \mathrm{~mm}$ for STN. Animals were allowed to recover from surgery for $9-15 \mathrm{~d}$ before starting the DBS treatment. The stimulation was delivered by a pulse generator/stimulator and a stimulus isolation unit (P2MP), which gave rectangular current pulses (monophasic). The delivered current was controlled daily using an oscilloscope (Hewlett Packard). DBS was applied continuously for $6 \mathrm{~d}$ at high frequency $(130 \mathrm{~Hz})$ on freely moving rats, except in one Pf group in which DBS was applied at the low frequency of $25 \mathrm{~Hz}$. The pulse width was set at $80 \mu \mathrm{s}$. Within the first minutes of HFS, the effects on the animal gross behavior of increasing progressively and briefly the stimulation intensity from 0 to $250 \mu \mathrm{A}$ were examined. Thereafter, the intensity was fixed at $80 \mu \mathrm{A}$ to avoid tissue damage with long-duration HFS. Animals were killed immediately after turning off the stimulation.

\section{Chronic L-DOPA treatment}

Twenty days after the 6-OHDA lesion, rats of the Pf-HFS group received chronic L-DOPA treatment for $21 \mathrm{~d}$. The treatment consisted of two injections per day ( $12 \mathrm{~h}$ interval) of $25 \mathrm{mg} / \mathrm{kg} \mathrm{L}$-DOPA and $12.5 \mathrm{mg} / \mathrm{kg}$ benserazide (Sigma-Aldrich) dissolved in $0.9 \% \mathrm{NaCl}$. HFS of Pf was applied all along the last $6 \mathrm{~d}$ of the L-DOPA treatment.

\section{Analysis of motor behavior}

Cylinder test. All groups of 6-OHDA-lesioned rats were scored for akinesia of the contralateral forelimb by using the cylinder test, in comparison with control rats. In brief, they were placed in a Plexiglas cylinder and, immediately after, videotaped for $30 \mathrm{~min}$ to examine the symmetry/ asymmetry of their forepaws use during their explorative behavior in this new environment. The numbers of contacts made on the cylinder wall during this period with the ipsilateral paw, the contralateral paw, and with both paws (double contacts) were determined and expressed as a percentage of the total number of contacts. In the Pf-HFS, Pf-LFS, and STN-HFS groups, the test was performed before starting and at the end of the stimulation period. For the L-DOPA + Pf-HFS group, testing was performed in the off-L-DOPA period, that is $12 \mathrm{~h}$ after the last injection of L-DOPA. Data are the means \pm SEM of the values determined from $n$ animals per group.

Corridor task. Sensorimotor neglect for the side contralateral to the lesion was also examined using the corridor task as described by Dowd et al. (2005). The food supply of animals was restricted to 15-17 g per day per individual to keep them at $85 \%$ of the free feeding weight. After habituation to the corridor (10 $\mathrm{min}$ in the corridor with scattered sugar pellets on the $2 \mathrm{~d}$ preceding the first test day, and $5 \mathrm{~min}$ in an empty corridor just before the test), animals were placed at an extremity of the corridor in which sugar pellets were available in small containers. Two versions (alternating and adjacent) of the test have been considered: in the alternating version, the containers were alternated along the left and right sides of the corridor at regular intervals; in the adjacent version, the containers were placed in pairs all along the corridor. Rats were left free to move in the corridor for $5 \mathrm{~min}$, during which they were videotaped. The numbers of retrievals made by the rat from containers on either side were counted, and the data were expressed as a percentage of the total number of retrievals. Data are the means \pm SEM of the values determined from $n$ animals per group. These two tests were performed for the Pf-HFS group. Because the results were equivalent between the two versions, only the alternating version was used for the STN-HFS group.

Abnormal involuntary movements score. The abnormal involuntary movements (AIMs) scores were used to evaluate LIDs. We use a modified version of the test described by Winkler et al. (2002). Animals were videotaped for $2 \mathrm{~h}$ after the L-DOPA injection, and axial, forelimb, and orolingual dyskinesias were scored from 0 to 4 for 1 min every $10 \mathrm{~min}$ in this time window (11 monitoring periods from 10 to $110 \mathrm{~min}$ postinjection). The animals were scored after 15 days of L-DOPA treatment and, a second time, at the end of Pf-HFS + L-DOPA treatment (day 21). 


\section{Morphological studies}

Tissue preparation. All animals were killed by decapitation after a 36-42 $\mathrm{d}$ postlesion survival time. The brains were quickly removed, then frozen in dry ice and stored at $-80^{\circ} \mathrm{C}$. Coronal $(10 \mu \mathrm{m}$ thick) tissue sections were cut at $-20^{\circ} \mathrm{C}$ with a cryostat (CM3050, Leica). The sections were then mounted on SuperFrost Plus glass slides (Fisher Scientific) and stored at $-80^{\circ} \mathrm{C}$ until specific treatment.

Histological control of 6-OHDA lesion and electrode placement. The loss of DA terminals in the striatum was assessed as an index of the extent of the DA denervation by analysis of $\left[{ }^{3} \mathrm{H}\right]$-mazindol binding to DA uptake sites, as described previously (Salin et al., 2002). Briefly, brain sections were air dried and rinsed for $5 \mathrm{~min}$ in $50 \mathrm{~mm}$ Tris buffer with $120 \mathrm{~mm} \mathrm{NaCl}$ and $5 \mathrm{~mm} \mathrm{KCl}$. They were then incubated for $40 \mathrm{~min}$ with $15 \mathrm{~nm}\left[{ }^{3} \mathrm{H}\right]-$ mazindol (NEN, DuPont; specific activity, $17 \mathrm{Ci} / \mathrm{mm}$ ) in $50 \mathrm{~mm}$ Tris buffer containing $300 \mathrm{~mm} \mathrm{NaCl}$ and $5 \mathrm{~mm} \mathrm{KCl}$ added with $0.3 \mathrm{~mm}$ desipramine to block the noradrenalin uptake sites. Sections were rinsed twice for $3 \mathrm{~min}$ in the Tris incubation buffer and for $10 \mathrm{~s}$ in distilled water and were air dried. ${ }^{3} \mathrm{H}$-sensitive photographic film (Kodak BioMax MS Film, Sigma) were apposed to the slides in $\mathrm{x}$-ray cassettes and exposed at room temperature for $21 \mathrm{~d}$. The levels of [ ${ }^{3} \mathrm{H}$ ]-mazindol labeling were quantified by digitized image analysis from the film autoradiograms using a BIOCOM analysis system (Densirag, BIOCOM). Gray levels were converted to optical densities (ODs) using external standards (calibrated density step tablet, Kodak). The mean OD value was determined from three sections per animal after subtracting the background signal measured on each section by scanning an area of the corpus callosum that is known to lack DA terminals.

The location of the stimulating electrode in the Pf and the STN was examined on toluidine blue-stained sections. Animals showing a reduction of $<85 \%$ in $\left[{ }^{3} \mathrm{H}\right]$-mazindol binding or a misplaced electrode were not included in the experimental groups presented above.

\section{In situ hybridization histochemistry}

Quantitative radioactive in situ hybridization was used to assess changes in intraneuronal mRNA levels of cytochrome oxidase subunit I (COI), as a general metabolic marker of neuronal activity, and of the neuropeptide precursors associated with the two populations of striatal projection neurons, preprodynorphin (PPDyn), and preprotachykinin (PPT), as markers of the direct pathway, and preproenkephalin (PPE) as a marker of the striatopallidal neurons, the first link of the indirect pathway. Probes were 43-48 mer synthetic oligonucleotides selected on the basis of the published sequence of PPE, PPDyn, PPT, and COI. Probes were 3 '-endlabeled by terminal deoxynucleotide transferase with ${ }^{35} \mathrm{~S}$-dATP $(1300$ $\mathrm{Ci} / \mathrm{mmol}$ ). The radiolabeled probes were then extracted on a mini Quick Spin Oligo Column (Roche).

All solutions used for in situ hybridization were treated with diethyl pyrocarbonate and autoclaved to avoid RNase degradation. Slidemounted sections were postfixed for $5 \mathrm{~min}$ in $3 \%$ paraformaldehyde. The sections were then incubated in prehybridization buffer containing $2 \times$ standard saline citrate (SSC). The sections were then acetylated for 10 min with $0.25 \%$ acetic anhydride in $0.1 \mathrm{M}$ triethanolamine and treated for $30 \mathrm{~min}$ in $0.1 \mathrm{M}$ Tris-glycine before being dehydrated in ethanol and air dried. Each section was covered with $35 \mu$ l of hybridization solution $(4 \times$ SSC with $50 \%$ formamide, $10 \%$ dextran sulfate, $1 \times$ Denhardt's solution, $0.25 \mathrm{mg} / \mathrm{ml}$ Escherichia coli tRNA, and $0.5 \mathrm{mg} / \mathrm{ml}$ sheared salmon sperm DNA) containing the radiolabeled probe (radioactivity level $\sim 500,000$ cpm per section), and incubated overnight at $47^{\circ} \mathrm{C}$ in humid chambers. Sections were then rapidly rinsed in ice-cold $2 \times$ SSC, then treated successively for 10 and $40 \mathrm{~min}$ with $1 \times$ SSC at room temperature, $1 \times$ SSC at $42^{\circ} \mathrm{C}$, and $0.1 \times$ SSC at $42^{\circ} \mathrm{C}$. Sections were then dehydrated in ethanol and air dried.

Sections were apposed to Kodak Bio-Max MR-1 film, and exposure time was adjusted to avoid film saturation. Those from control and experimental groups of animals were run together in the same experimental session and exposed side by side on the same autoradiographic film. Sections processed for cellular analysis of COI mRNA levels were thereafter coated with GE Healthcare LM1 autoradiographic emulsion and exposed at $4^{\circ} \mathrm{C}$ for $1-3$ weeks. Exposed slides were developed in Kodak $\mathrm{D}-19$ for $4 \mathrm{~min}$ at $13^{\circ} \mathrm{C}$ and counterstained with toluidine blue.

\section{Data analysis}

Levels of autoradiographic labeling were quantified by a computerized imaging system (BIOCOM). Analysis of striatal PPE, PPDyn, and PPT was performed from autoradiographic films, and was restricted to the dorsal part of the structure, excluding the nucleus accumbens. Gray levels were converted to relative OD by using standard internal curves. The background signal was determined for each section by scanning the corpus callosum and subtracted from values obtained in the striatum on the same section. The mean OD was determined from three sections of each animal.

Analysis of COI mRNA labeling was performed at the cellular level on emulsion-coated sections in the GP, EP, STN, and substantia nigra pars reticulate $(\mathrm{SNr})$. Sections were observed under dark-field epilumination with an immersion $20 \times$ objective lens of a microscope connected to a Cohu camera, and the digitized images were transferred to the screen of a video monitor with a resulting magnification of 1000 . Using the Visioscan image analysis software from BIOCOM, the number of silver grains per cell was estimated under polarized light by measuring OD with respect to a standard curve of a defined number of silver grains. Specific labeling was determined after subtracting autoradiographic background on the same section. A sampling of at least 50 labeled neurons $(>10$ grains) per section was quantified in three sections from each animal, and the mean number of silver grains per neuron determined. For both film and cellular analyses, the data from all the animals per condition were averaged and were expressed as the percentage \pm SEM of the corresponding control values.

For cellular and behavioral studies, statistical analyses of data were performed using a one-way ANOVA followed by a Student-NewmanKeuls test for multiple group comparison. To compare the same rats in different conditions (before and after stimulation), a paired $t$ test was performed. A significance of $p<0.05$ was required for rejection of the null hypothesis.

\section{Results}

\section{Control of the electrode location in Pf and of 6-OHDA-induced denervation extent in the striatum}

Figure 1 illustrates the location of the stimulating electrode, which was in the dorsolateral part of the Pf in the selected animals. No major tissue damage was observed after $6 \mathrm{~d}$ of continuous stimulation.

The animals with or without Pf-HFS that received a unilateral injection of 6-OHDA showed an almost complete loss of $\left[{ }^{3} \mathrm{H}\right]$ mazindol binding in the ipsilateral striatum (Fig. 2). No significant change of the striatal $\left[{ }^{3} \mathrm{H}\right]$-mazindol binding was found in the side contralateral to the lesion.

\section{Behavioral observations}

Pf-DBS was applied at a fixed low intensity $(80 \mu \mathrm{A})$ all along the $6 \mathrm{~d}$ stimulation period to avoid tissue damage with prolonged application. However, within the 2 min before fixing the stimulation intensity, we examined whether HFS of Pf at increasing intensities can induce per se abnormal involuntary movements as observed for STN-HFS (Salin et al., 2002; Bacci et al., 2004; Boulet et al., 2006; Oueslati et al., 2007). Whereas STN-HFS elicited abnormal involuntary movement from $\sim 110 \mu \mathrm{A}$ in our experimental conditions, such movements were never induced by HFS of Pf for intensities up to $250 \mu \mathrm{A}$. No alteration of the animal gross behavior was observed.

\section{Cylinder test}

In these experiments, the efficiency of HFS of Pf and of STN to alleviate akinesia was compared. Control rats $(n=8)$ made a majority of double contacts $(58.6 \pm 4.4)$ on the cylinder wall during their explorative behavior. The three groups of hemiparkinsonian rats, unimplanted $(n=10)$ and implanted for Pf-HFS $(n=10)$ or STN-HFS $(n=8)$ in prestimulation conditions, all 
A

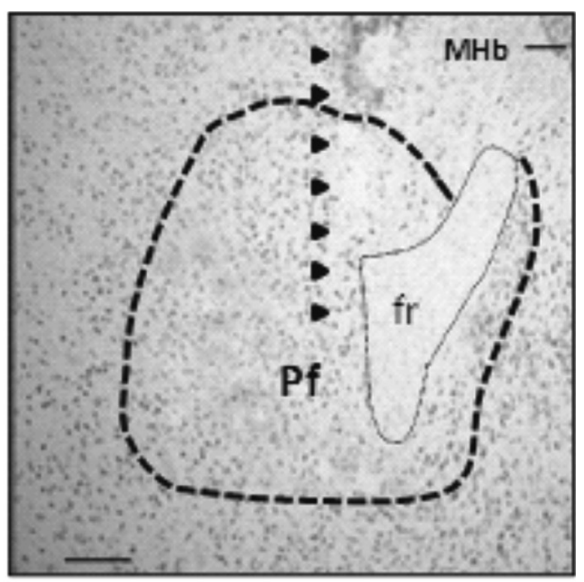

B

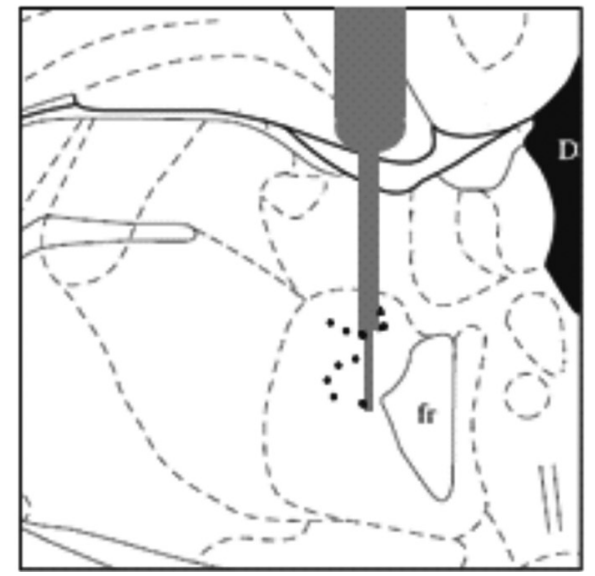

Figure 1. Location of the stimulating electrode. $A$, Photomicrograph of a toluidine bluestained section at $P f$ nucleus level (delineated by dotted line) illustrating the implantation site (black arrows). B, Schematic diagram adapted from the stereotaxic atlas of Paxinos and Watson (1986) showing the location of the electrode tips (black points) for all stimulated animals ( $n=$ 10). fr, Fasciculus retroflexus; MHb, medial habenular nuclei. Scale bar, $150 \mu \mathrm{m}$.

showed a dramatic decrease in the proportion of double contacts due to decreased spontaneous use of the contralateral forepaw $(-86 \%,-89.5 \%$, and $-83 \%$, respectively; $p<0.01)$. No significant differences were found among these lesioned groups, suggesting that the electrode implantation alone does not affect the scores. HFS of both Pf and STN efficiently alleviated this deficit, as evidenced by the increase in the number of double contacts compared with the 6-OHDA group $(+271 \%$ and $+450 \%$, respectively; $p<0.01$ ) or to respective prestimulation values. Reversal was partial, the scores remaining significantly decreased versus controls by $-26.6 \%(p<0.01)$ after STN-HFS and $-50.5 \%(p<0.01)$ after Pf-HFS. A significant difference was found between the two stimulated groups, showing that Pf-HFS is less potent than STN-HFS $(p<0.01)$. (Fig. 3). Pf-LFS did not induce any relief of the deficit triggered by the dopamine lesion ( $-85.7 \%$ compared with controls; $-0.24 \%$ compared with the 6-OHDA group).

\section{Corridor test}

Control animals $(n=8)$ performed an equivalent number of retrievals from each side of the corridor $(\sim 50 \%$ of the total retrievals from either side) in the alternating or the adjacent versions of the corridor. Animals with the unilateral 6-OHDA lesion $(n=10)$ exhibited a strong bias toward the side ipsilateral to the lesion: they made $84 \%$ and $90 \%$ of retrievals from the ipsilateral side in the two versions $(+74 \%$ vs controls in alternating corri-
A
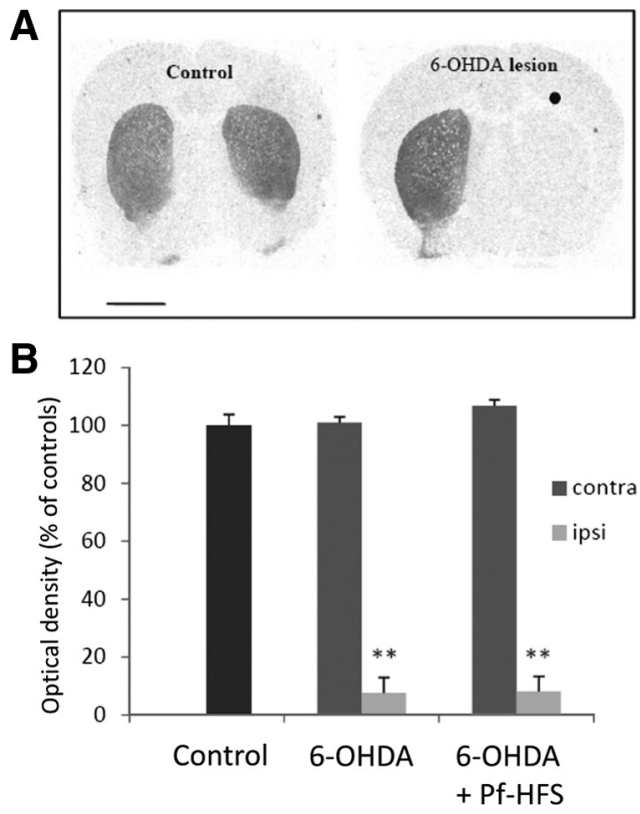

Figure 2. $\quad \boldsymbol{A}, \boldsymbol{B}$, Digitized autoradiographic images $(\boldsymbol{A})$ and quantitative analysis $(\boldsymbol{B})$ showing the effects of unilateral 6 -hydroxydopamine lesion on striatal [ $\left.{ }^{3} \mathrm{H}\right]$-mazindol binding to dopamine uptake sites. The data presented in the graphs are the means \pm SEM of the optical density values determined from $n$ animals per condition and are expressed as percentages of controls. Statistical comparison was performed using a one-way ANOVA test followed by Student-Newman-Keuls test. Scale bar, $2 \mathrm{~mm}$. @, Side ipsilateral to surgery. ${ }^{* *} p<0.01$ compared with control values.

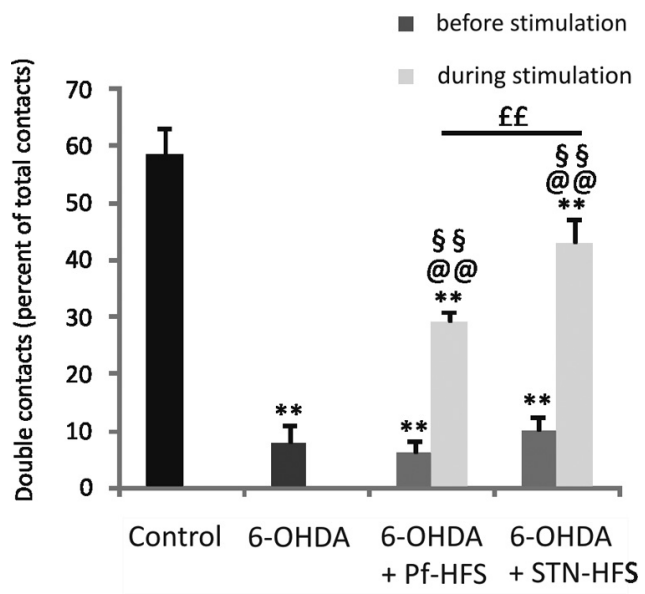

Figure 3. Effects of STN-HFS or Pf-HFS on 6-OHDA lesion-induced akinesia evaluated using the cylinder test. The data presented in the graphs are the means \pm SEM of the double contacts on the wall of the cylinder, expressed as the percentage of the total number of contacts, determined from $n$ animals. Statistical comparison was performed using a one-way ANOVA followed by Student-Newman-Keuls test for multiple group comparison. The numbers of animals per group were as follows: 8 controls, $106-0 \mathrm{HDA}, 10$ Pf-HFS, and 8 STN-HFS. ${ }^{* *} p<0.01$ compared with control values; ${ }^{@} p<0.01$ compared with 6-OHDA lesion; ${ }^{\mathrm{EE}} p<0.01$ comparing STN-HFS and Pf-HFS. In addition, a paired Student's $t$ test is used to compare the scores of the same rats before or during HFS. ${ }^{\S \S} p<$ 0.01 compared with prestimulation.

dor, $p<0.01 ;+76 \%$ vs controls in adjacent corridor, $p<0.01$ ), neglecting food on the contralateral side $(-68 \%$ vs controls in alternating corridor, $p<0.01)$. Pf-HFS $(n=6)$ or STN-HFS ( $n=$ 4) completely reversed this bias, with the animals recovering the ability to make $\sim 50 \%$ of food retrievals from the side contralateral to the lesion $(p<0.01)$ (Figs. 4,5$)$. 
A

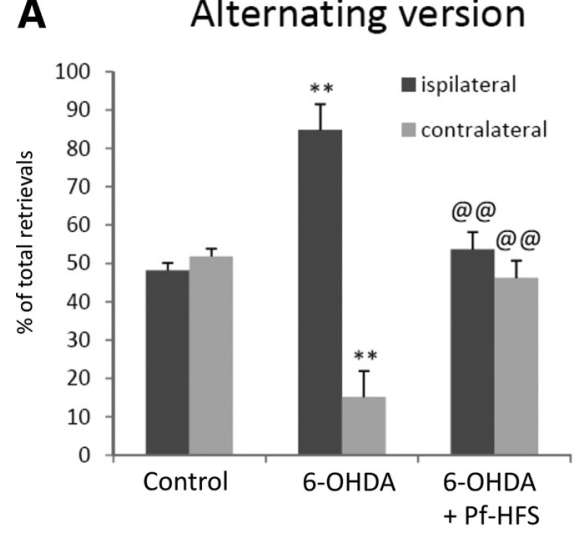

B Adjacent version

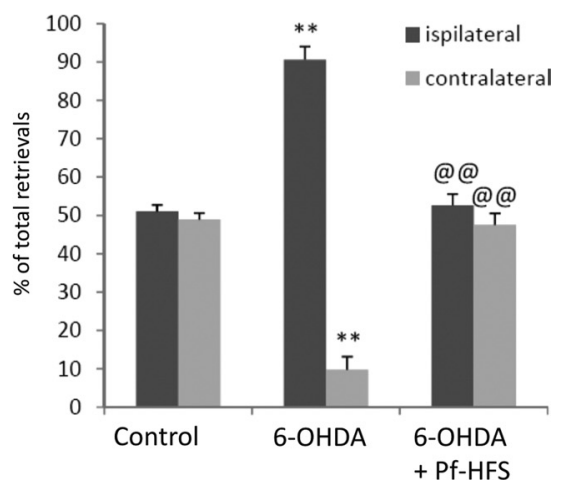

Figure 4. $\quad \boldsymbol{A}, \boldsymbol{B}$, Effect of unilateral 6-hydroxydopamine lesion followed or not by Pf-HFS on sensorimotor neglect assessed with the alternating $(\boldsymbol{A})$ or adjacent version $(\boldsymbol{B})$ of the corridor test. The data presented in the graphs are the means \pm SEM of the retrievals from either side of the body, expressed as the percentage of total retrievals determined from $n$ animals per group. Statistical comparison was performed using a one-way ANOVA followed by Student-Newman-Keuls test. The numbers of animals per group were as follows: 8 controls, $106-0$ HDA, and 6 Pf-HFS. ${ }^{* *} p<0.01$ compared with control values; ${ }^{@} p<0.01$ compared with 6-OHDA lesion values.

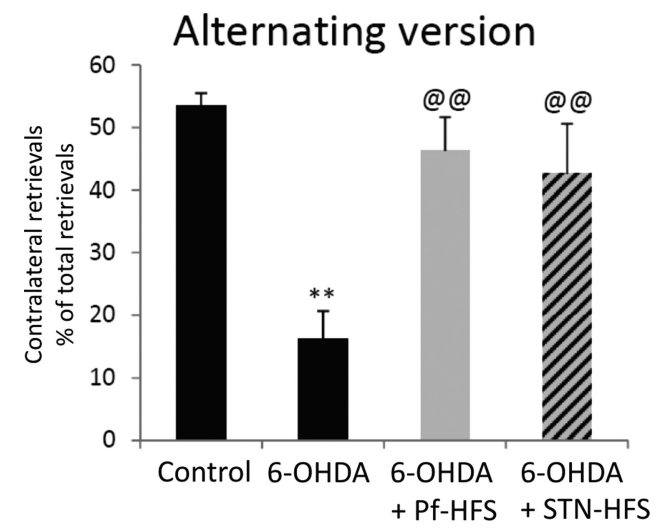

Figure 5. Effect of STN-HFS or Pf-HFS on 6-OHDA lesion-induced sensorimotor neglect, assessed with the alternative version of the corridor test. The data presented in the graphs are the means \pm SEM of the contralateral retrievals expressed as percentages of total retrievals determined from $n$ animals. Statistical comparison was performed using a one-way ANOVA followed by Student-Newman-Keuls test. The numbers of animals per group were as follows: 8 controls, $106-0$ HDA, 6 Pf-HFS, and 4STN-HFS. ${ }^{* *} p<0.01$, compared with control values; ${ }^{@} p<0.01$ compared with 6-0HDA lesion values.

Interaction between Pf-HFS and L-DOPA

To assess the ability of Pf-HFS to relieve LIDs, we measured AIMs of L-DOPA-treated rats $(n=5)$ before starting Pf-HFS (at day 15 of L-DOPA treatment) and the last day of HFS (day 21 of L-DOPA treatment). Animals presented almost no axial dyskinesias (grades $0-1$; mean score, $0.5 \pm 0.2$ ), occasional to moderate orofacial dyskinesias (grades 1-2; mean score, $1.2 \pm 0.2$ ), and moderate to severe forepaw dyskinesias (grades 2-4; mean score, $2.8 \pm 0.4)$. After $6 \mathrm{~d}$ of Pf-HFS, the mean score of forelimb dyskinesias was significantly reduced by $28.6 \%(p<0.05)$ compared with prestimulation (grades $1-3$; mean score, $2 \pm 0.4$ ) (Fig. $6 A$ ). The orofacial and axial dyskinesia scores were also reduced $(0.7 \pm$ 0.2 and $0.2 \pm 0.1$, respectively), but no valid statistical comparison could be made with such narrow ranges of values.

When testing the same Pf-HFS + L-DOPA-treated animals in the cylinder test, in the off-L-DOPA period ( $12 \mathrm{~h}$ after the last injection of L-DOPA) no more beneficial anti-akinetic effect of the stimulation was observed: the number of double contacts was decreased compared with animals with Pf-HFS alone $(-98 \%, p<$

$0.01)$ and did not statistically differ from the 6-OHDA group (Fig. 6B). Also in the corridor test, in contrast to animals treated by Pf-HFS only, the Pf-HFS + L-DOPAtreated animals did not recover from the contralateral neglect: the contralateral scores were significantly reduced versus controls and Pf-HFS alone, and were no more different from the untreated 6-OHDA group (Fig. 6C). These data point to strong interferences between the two treatments.

Effects of Pf-HFS on the mRNA levels of striatal neuropeptide precursors

The results presented concern the dopaminedepleted striatum. No significant changes were measured in the striatum contralateral to surgery in the experimental conditions examined.

\section{Preproenkephalin A mRNA levels}

The expression of PPE was increased by $101.6 \%$ in the 6-OHDA group compared with controls $(p<0.01)$ (Fig. $7 A, B)$. Animals with the 6-OHDA lesion that received Pf-HFS present a significant reduction of PPE mRNAs levels in comparison with animals with the 6-OHDA lesion alone $(-19.25 \%, p<0.05)$. However, the expression of PPE remained significantly increased versus control animals $(+62.7 \%, p<0.01)$, showing that reversal of the dopamine lesion-mediated response by Pf-HFS was partial.

Preprodynorphin mRNA levels

In the 6-OHDA group, PPDyn mRNA levels were decreased compared with controls $(-32.4 \%, p<0.05)$ (Fig. 8). In the PfHFS group, PPDyn mRNA levels did not significantly differ from the 6-OHDA group and remained decreased compared with controls $(-22.35 \%$ vs controls, $p<0.05)$.

\section{Preprotachykinin $m R N A$ levels}

PPT mRNA expression was decreased by $56.94 \%$ in $6-\mathrm{OHDA}$ animals compared with controls $(p<0.01)$ (Fig. 8). This response was not significantly modified by Pf-HFS $(+16.76 \%$ vs 6 -OHDA lesion animals; $-49.72 \%$ vs control group, $p<0.01$ ).

\section{COI mRNAs levels in the globus pallidus and in the} subthalamic nucleus

Compared with control animals, the dopamine denervation induced a significant increase in COI mRNA levels in the GP and the STN ipsilateral to the lesion $[38.7 \%(p<0.01)$ and $36.8 \%$ $(p<0.01)$, respectively] (Fig. 9A,B). Pf-HFS totally reversed this increase both in GP and in STN, with the values being significantly decreased compared with 6-OHDA alone (GP: $-20.8 \%$, $p<0.05$; STN: $-32.8 \%, p<0.01)$ and no more different from controls. No significant differences in COI mRNA levels were measured in these structures in the side contralateral to the lesion in the different conditions examined, although a tendency toward decrease was observed in the STN of the Pf-HFS group compared with controls or 6-OHDA group alone (Fig. 9).

COI mRNAs levels in the entopeduncular nucleus and in the substantia nigra pars reticulata

Animals with the 6-OHDA lesion alone exhibited a significant increase in COI mRNAs in the EP and the SNr ipsilateral to the lesion $[51.7 \%(p<0.01)$ and $38.5 \%(p<0.01)$, respectively] 

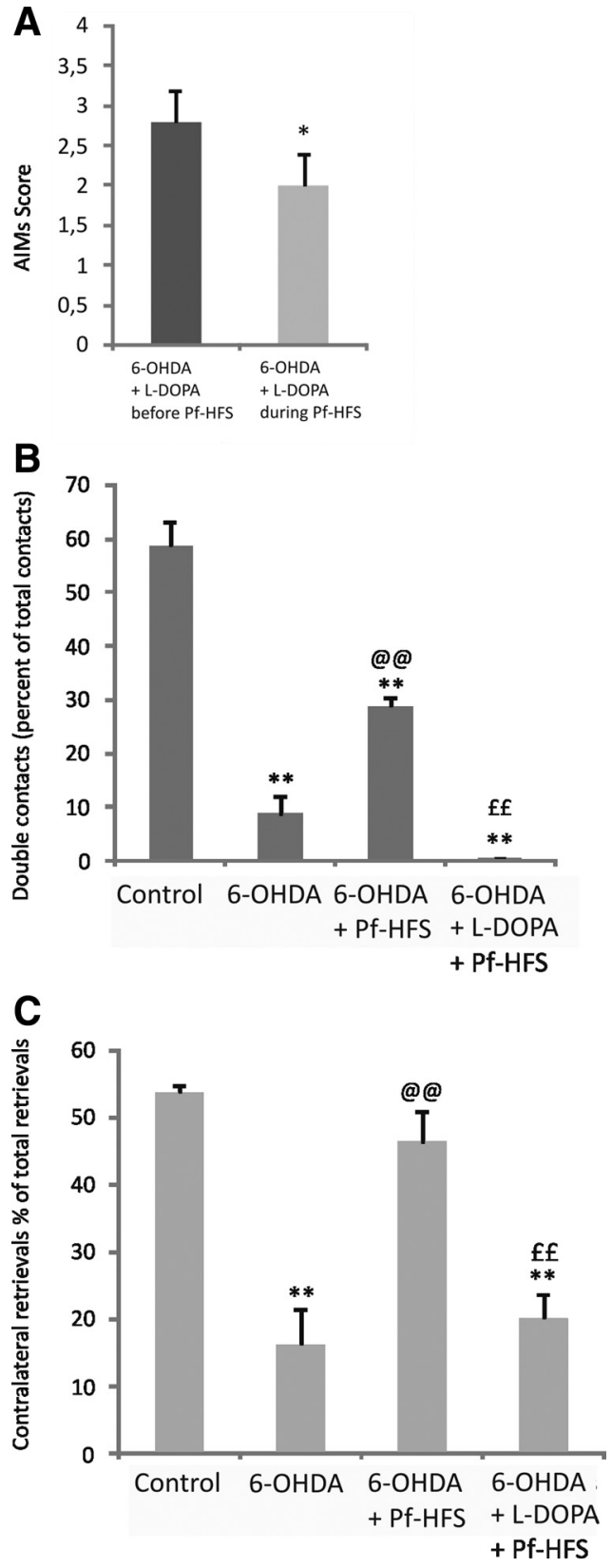

Figure 6. $\quad A-C$, Effect of combined chronic L-DOPA treatment and Pf-HFS on L-DOPA-induced forepaw dyskinesia $(\boldsymbol{A})$, on 6-0HDA lesion-induced akinesia $(\boldsymbol{B})$, or on sensorimotor neglect induced by 6-OHDA lesion using the corridor test (C). In $A$, the scores of forepaw dyskinesias were assessed at day 15 of the chronic L-DOPA treatment before starting Pf-HFS, and at day 21 of $\mathrm{L}-\mathrm{DOPA}$ treatment and $6 \mathrm{~d}$ of stimulation. The data are the mean of the scores determined during 1 min observation every 10 min from 10 to 110 min after the L-DOPA injection. Statistical comparison was performed using a $t$ test to compare the scores of the same rats before or during HFS. In $B$ and $C$, behavioral assessments were performed $12 \mathrm{~h}$ after the last injection of L-DOPA at day 21 of the chronic treatment. Statistical comparison was performed using a one-way ANOVA followed by Student-Newman-Keuls test. The numbers of animals per group were as follows: 8 controls, $106-$ OHDA, 10 Pf-HFS, and 5 Pf-HFS + L-DOPA. ${ }^{* *} p<0.01$ and ${ }^{*} p<0.05$ compared with before stimulation $(A)$ or control values $(B, C) ;{ }^{@} p<0.01$ compared with 6-OHDA lesion; and ${ }^{\mathrm{ff}} p<0.01$ compared with Pf-HFS values $(\boldsymbol{B}, \boldsymbol{C})$.

(Fig. 10A, B) compared with control animals. After Pf-HFS, COI mRNA levels were significantly reduced in EP versus 6-OHDA lesion $(-18.69 \%$; $p<0.05)$ but remained increased versus controls $(+23.34 \%, p<0.05)$. In SNr, Pf-HFS reversed the lesioninduced increase, with COI mRNA levels being significantly reduced versus 6 -OHDA $(-18.79 \%$; $p<0.05)$ and being no
A

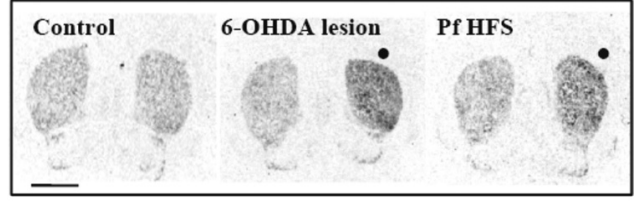

B

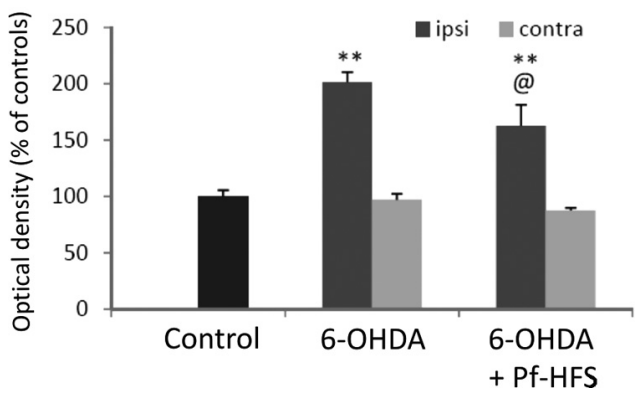

Figure 7. $\quad \boldsymbol{A}, \boldsymbol{B}$, Digitized autoradiographic images $(\boldsymbol{A})$ and quantitative analysis $(\boldsymbol{B})$ showing the effects of separate or combined unilateral 6-hydroxydopamine lesion and Pf-HFS on striatal PPE mRNA expression. The data presented in the graphs are the means \pm SEM of the optical density values determined from $n$ animals per condition and are expressed as the percentages of control. Statistical comparison was performed using a one-way ANOVA followed by StudentNewman-Keuls test. Scale bar, 2 mm. Side ipsilateral to surgery. ${ }^{* *} p<0.01$ compared with control values; ${ }^{@} p<0.05$ compared with 6-0HDA lesion values.

more different from controls. No significant differences in COI mRNA levels (Fig. 10) were detected between animals with dopamine lesion alone and those with the lesion and Pf-HFS in the side contralateral to the lesion.

\section{Discussion}

This study shows that Pf-HFS efficiently alleviates akinesia and sensorimotor neglect in a PD model based on extensive dopamine lesion. This anti-parkinsonian effect is associated with partial reversal of the increase in striatal PPE expression; a complete suppression of the dopamine lesion-induced metabolic changes in the STN, the GP, and the SNr; and a partial reduction of these changes in EP. Compared with previous data about STN-HFS in similar conditions (Lacombe et al., 2009), Pf-HFS appears to have more widespread action on the BG network, with additional impact on striatal neurons of the indirect pathway and on EP (Table 1). These data highlight subcircuits involved by Pf-HFS that may be distinct from those affected by STN-HFS and provide the first cellular substrates for the therapeutic effects mediated by $\mathrm{CM} / \mathrm{Pf}$ DBS (Caparros-Lefebvre et al., 1999; Krauss et al., 2002; Mazzone et al., 2006; Peppe et al., 2008; Stefani et al., 2009). In addition, complex interactions between Pf-HFS and L-DOPA treatment are evidenced: the neurosurgical treatment reduces LIDs, and, conversely, L-DOPA treatment interferes with the antiparkinsonian efficiency of Pf-HFS.

\section{Pf and movement abnormalities in PD state}

Effects of Pf-HFS versus lesion

It is now recognized that the action mechanisms of HFS are more complex than modulation of the activity of neurons in the targeted structure and might involve adaptive changes in large neuronal networks. In particular, a recent study using optogenetics has shown that the anti-parkinsonian action of STNHFS is linked rather to activation of afferent input systems to STN than to inactivation of STN neurons (Gradinaru et al., 2009). Therefore, the effects of lesions and HFS might in some 
A

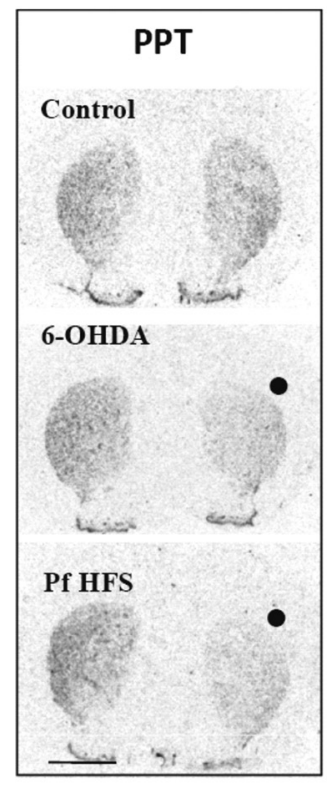

Figure 8. $\quad \boldsymbol{A}, \boldsymbol{B}$, Digitized autoradiographic images $(\boldsymbol{A})$ and quantitative analysis $(\boldsymbol{B})$ showing the effects of separate or combined unilateral 6-hydroxydopamine lesion and Pf-HFS on striatal PPT and PPDyn mRNA expression. The data presented in the graphs are the means \pm SEM of the optical density values determined from $n$ animals per condition and are expressed as percentages of controls. Statistical comparison was performed using a one-way ANOVA followed by Student-Newman-Keuls test. Scale bar, 2 mm., Side ipsilateral to surgery. ${ }^{* *} p<0.01$ and ${ }^{*} p<0.05$ compared with control values.

A
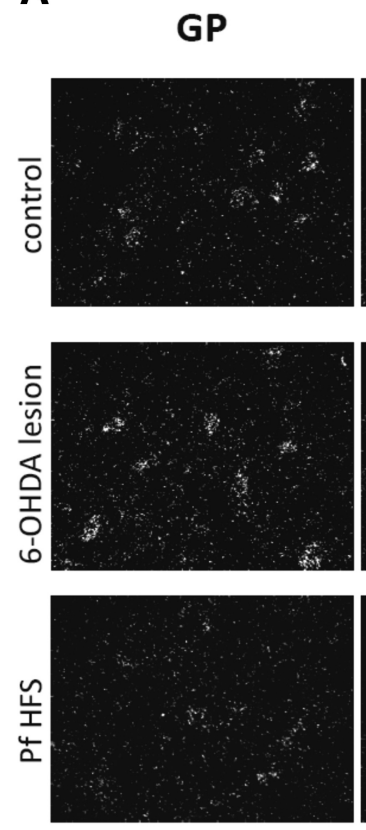

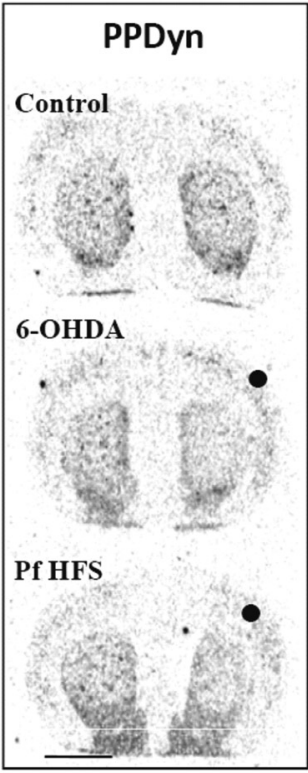

B
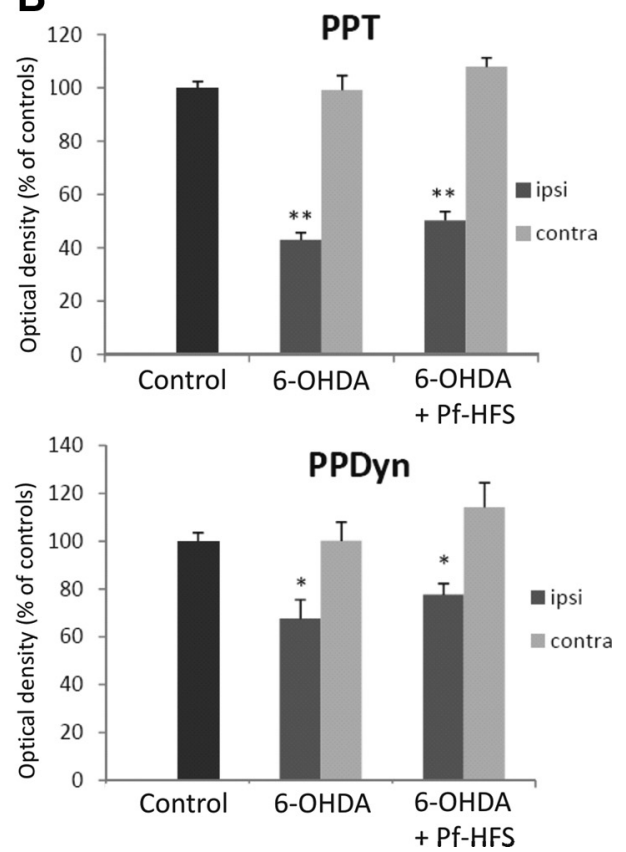

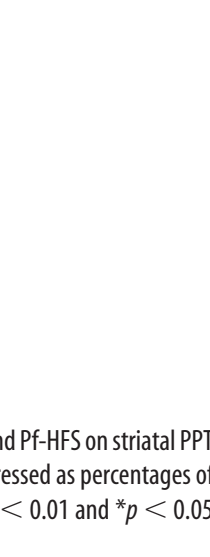
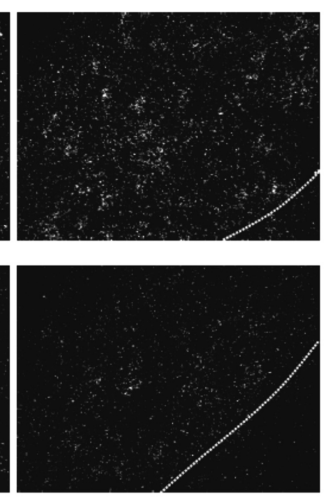

B GP
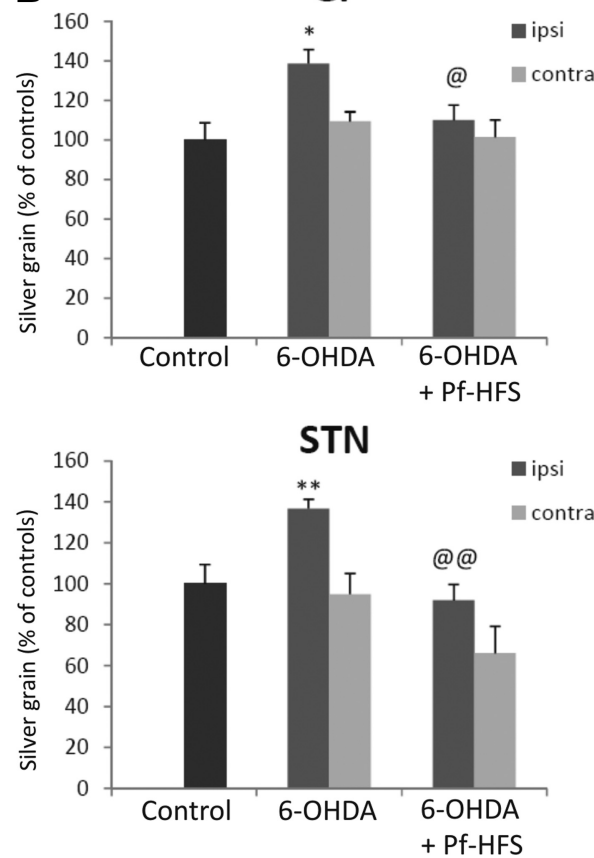

Figure 9. $\boldsymbol{A}, \boldsymbol{B}$, Photomicrographs $(\boldsymbol{A})$ and quantitative analysis $(\boldsymbol{B})$ showing the effects of separate or combined unilateral 6-hydroxydopamine lesion and Pf-HFS on COI mRNA expression in GP and STN. Sections were processed for in situ hybridization with ${ }^{35}$ S-radiolabeled COl oligonucleotide probe and emulsion autoradiography. The data presented in the graphs are the means \pm SEM of silver grain numbers determined from $n$ animals per condition and are expressed as percentages of controls. Statistical comparison was performed using a one-way ANOVA followed by Student-Newman-Keuls test. Scale bar, $20 \mu \mathrm{m} .{ }^{* *} p<0.01$ and ${ }^{*} p<0.05$ compared with control values; ${ }^{@} p<0.01$ and ${ }^{@} p<0.05$ compared with 6-0HDA lesion values. cp, Cerebral peduncle.

instances differ. We previously reported, using the same rat model of PD, that Pf lesion, as shown here for Pf-HFS, counteracts a great part of the effects of the dopamine lesion on gene expression of markers of neuronal activity in the BG (Bacci et al., 2004). The major difference concerns the output structures of the BG: Pf-HFS reverses the lesion-induced changes in COI mRNA levels totally in SNr and partially in EP whereas Pf lesion reversed the changes in GAD67 mRNA levels, a metabolic marker of GABA neurons, in EP but not SNr. In addition, the dopamine lesion-induced increase in striatal 
A
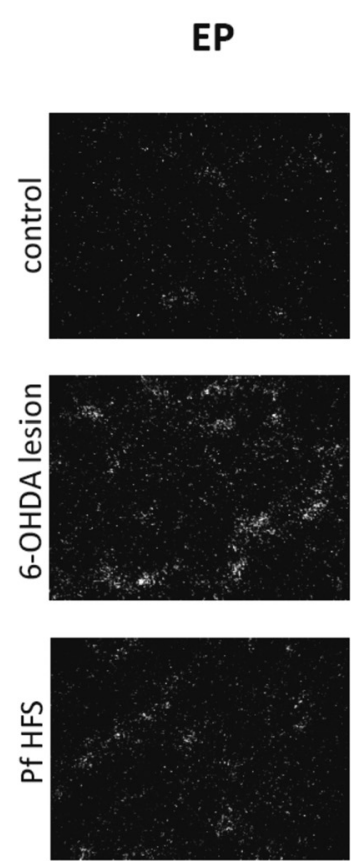
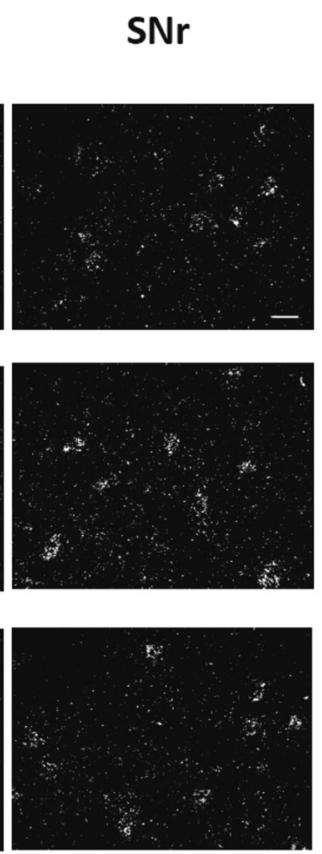

B
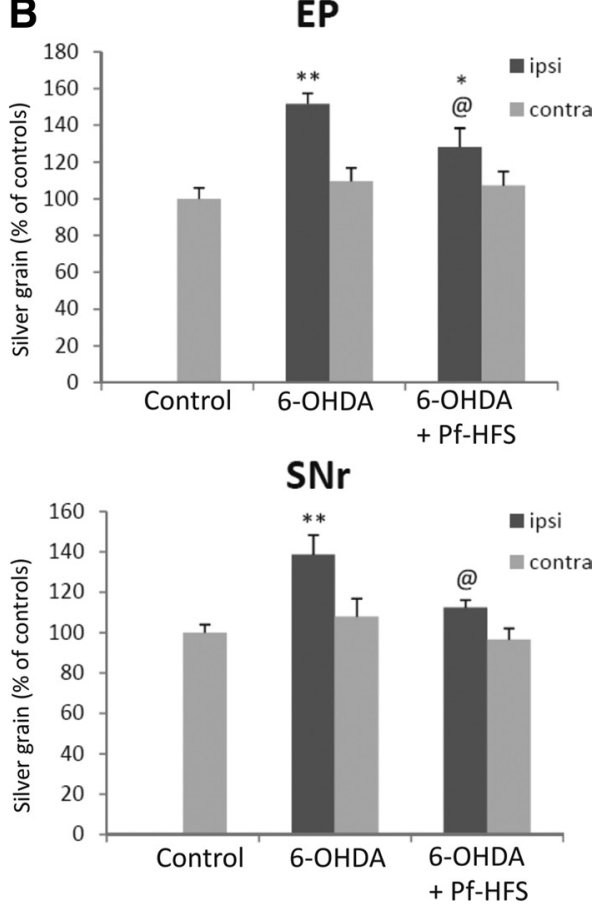

Figure 10. $\quad \boldsymbol{A}, \boldsymbol{B}$, Photomicrographs $(\boldsymbol{A})$ and quantitative analysis $(\boldsymbol{B})$ showing the effects of separate or combined unilateral 6-hydroxydopamine lesion and Pf-HFS on COI mRNA expression in EP and SNr. Sections were processed for in situ hybridization with ${ }^{35}$ S-radiolabeled COl oligonucleotide probe and emulsion autoradiography. The data presented in the graphs are the means \pm SEM of silver grain number determined from $n$ animals per condition and are expressed as percentages of controls. Statistical comparison was performed using a one-way ANOVA followed by Student-Newman-Keuls test. Scale bar, $20 \mu \mathrm{m} .{ }^{* *} p<0.01$ and ${ }^{*} p<0.05$ compared with control values; ${ }^{\circledR} p<0.05$ compared with 6-0HDA lesion values.

Table 1. Impact of Pf-HFS or STN-HFS on the cellular alterations induced by the dopamine denervation in the basal ganglia

\begin{tabular}{llllllll}
\hline & Striatum & & & GP & STN & EP & SNr \\
\cline { 2 - 7 } & PPDyn & PPT & PPE & COI & COI & COI & COI \\
\hline 6-OHDA lesion & $\downarrow$ & $\downarrow$ & $\uparrow$ & $\uparrow$ & $\uparrow$ & $\uparrow$ & $\uparrow$ \\
Pf-HFS & $\downarrow$ & $\downarrow$ & $\uparrow^{*}$ & $\leftrightarrow^{*}$ & $\leftrightarrow^{*}$ & $\uparrow^{*}$ & $\leftrightarrow^{*}$ \\
STN-HFS & $\downarrow$ & $\downarrow$ & $\uparrow$ & $\leftrightarrow^{*}$ & $\leftrightarrow^{*}$ & $\uparrow$ & $\leftrightarrow^{*}$ \\
\hline
\end{tabular}

Summary table comparing the impact of Pf-HFS and STN-HFS on the cellular alterations induced by the dopamine denervation in the basal ganglia. $\uparrow$, Increase; $\downarrow$, decrease; $\leftrightarrow$, normalization as compared to control; ${ }^{*}$, statistically different from 6-OHDA lesion values.

PPE mRNA levels is partially reversed by Pf-HFS, but totally by Pf lesion. Whether these cellular differences translate into a differential impact on parkinsonian deficits is a matter of concern. We show here that Pf-HFS allows significant, although partial, recovery of the impaired spontaneous use of the contralateral forepaw induced by unilateral dopamine lesion in the cylinder test. This provides evidence for efficient anti-akinetic action of Pf-HFS, and complies with a clinical report showing that Pf-HFS ameliorates extrapyramidal symptoms (Stefani et al., 2009). There are no data available about the anti-parkinsonian action of the Pf lesion in the rat, but a recent study showed no significant persistent motor improvement after CM/Pf lesion in the MPTP monkey model of PD (Lanciego et al., 2008). This lack of effect could be due to the fact that the CM/Pf lesion was performed unilaterally in a bilateral dopaminergic lesion model, whereas crossed projections from $\mathrm{CM} / \mathrm{Pf}$ to $\mathrm{STN}$ and $\mathrm{SNr}$ have been reported (Marini et al., 1999; Castle et al., 2005). Another explanation is that reversal of the response to the dopamine lesion in $\mathrm{SNr}$ is a requisite for efficient beneficial action on forelimb akinesia. Indeed, evidence has been provided in parkinsonian monkeys that $\mathrm{SNr}$ is involved in the development of parkinsonian signs, particularly those affecting appendicular function (Wichmann et al., 2001).

\section{Effects of Pf-HFS versus STN-HFS}

In compliance with recent clinical data (Stefani et al., 2009), we found that Pf-HFS is less potent than STN-HFS in alleviating akinesia. It is of interest to compare the impact of Pf-HFS on the pathophysiological functioning of the BG with previous data on STN-HFS (Oueslati et al., 2007; Lacombe et al., 2009). HFS of both targets totally reverses the metabolic changes produced by the dopamine lesion in the SNr in addition to GP and STN, and does not interfere with the postlesional modifications in the striatal neurons of the direct pathway. But, the effects differ at the level of the EP and striatal PPE-expressing neurons: Pf-HFS partially reverses the effects of the dopamine lesion, whereas STNHFS has no effect. Together, the comparisons of Pf-HFS with Pf lesion and with STN-HFS reveal a more marked anti-akinetic effect in conditions where metabolic activity is normalized in $\mathrm{SNr}$ but not in EP and the striatal indirect pathway. It then could be that reversing the effect of the dopamine lesion in $\mathrm{SNr}$ has an anti-akinetic effect, whereas reversal in EP may have rather a pro-akinetic effect. In this connection, we also show here that Pf-HFS relieves forelimb dyskinesias induced by chronic L-DOPA treatment, whereas STN-HFS exacerbates this side effect. Also, clinical data have reported that CM/Pf-HFS may be helpful for the management of LIDs (Caparros-Lefebvre et al., 1999; Stefani et al., 2009), and it is conceivable that the strong connections between CM/Pf and GPi/EP (Sidibé et al., 2002) are responsible both for the lower efficiency of Pf-HFS in alleviating akinesia and for its direct anti-dyskinetic action compared with STN.

\section{Pf-HFS and sensorimotor neglect}

Some abnormalities in sensorimotor processing have already been reported in PD patients (Berardelli et al., 2001). First described in rats with lateral hypothalamus lesion (Marshall et al., 
1974), which damages ascending dopaminergic systems, the phenomenon of lateralized neglect is characteristic of rats with unilateral dopaminergic denervation. Initially considered as sensorimotor in nature, this deficit might primarily reflect an impairment of the initiation of actions directed toward contralateral events (Carli et al., 1985). Based on this phenomenon, the corridor task has been validated as a good behavioral test for assessing the deficit of sensorimotor integration induced by the nigrostriatal dopamine lesion and the recovery by dopamine receptor agonist or transplantation of fetal dopaminergic neurons in hemiparkinsonian rats (Dowd et al., 2005; Fitzsimmons et al., 2006). Here, we demonstrate that this task depends not only on dopamine tone, but is also useful to evaluate nondopaminergic anti-parkinsonian therapies. Pf-HFS completely reversed the neglect toward the contralateral side induced by the unilateral 6-OHDA lesion, re-establishing the balance of food retrievals from both sides of the body. The potent action of Pf-HFS in this test of lateralized response selection is to be considered in particular in relationship with the proposed role of the CM/Pf-striatal system in the modulation of motor responses under behaviorally significant external stimuli and as the entrance of information with attentional orienting value to the BG circuits (Matsumoto et al., 2001; Van der Werf et al., 2002; Raeva, 2006; Minamimoto et al., 2009). On the other hand, we also examined the impact of STN-HFS in the corridor task and observed a beneficial effect equivalent to that provided by Pf-HFS, whereas the two surgical treatments have differential qualitative or quantitative outcomes on akinesia or dyskinesias. It could be suggested that lateralized neglect preferentially involves the BG subcircuits that show common responses to HFS of the two targets (Table 1) rather than the $\mathrm{EP}$ and/or the PPE-expressing striatal neurons.

\section{Interaction between L-DOPA treatment and Pf-HFS}

Treatments with dopamine agonists or L-DOPA are known to relieve the ipsilateral bias created by the dopamine lesion in the corridor task as well as akinesia in the cylinder test. However, LIDs can affect the performance in chronic treatment conditions (Lundblad et al., 2002). For this reason, the effects of the combined treatment with Pf-HFS and L-DOPA were assessed $12 \mathrm{~h}$ after L-DOPA administration, thus at off condition. We surprisingly found that Pf-HFS is much less efficient in alleviating limb akinesia and lateralized neglect when applied in animals with chronic L-DOPA treatment than when applied alone, suggesting that the beneficial effects of Pf-HFS might be compromised by L-DOPA treatment. Such interaction may contribute to minimize the anti-parkinsonian efficiency of CM/Pf-HFS in the PD state and account for the weak benefits on UPDRS scores that have been reported from a small cohort of patients (Stefani et al., 2009). Together with the above observation that Pf-HFS alleviates forelimb dyskinesias induced by chronic L-DOPA, these data point to complex interactions between the two treatments, and further between CM/Pf and dopamine tone in the BG, which might be taken into consideration in the indication of $\mathrm{CM} / \mathrm{Pf}$ DBS in the management of movement disorders.

\section{Conclusion}

This study demonstrates that CM/Pf is a key node for modulating BG functioning, supporting the increasing interest for this complex in the surgical treatment of BG-related disorders. Our findings confirm and extend the clinical data showing moderate impact of Pf-HFS on extrapyramidal PD symptoms and LIDs but a marked outcome on more integrative deficits. They point to the preferential relationships between Pf and EP as a substrate for the different, qualitative or quantitative, impact of HFS of the Pf versus STN on motor dysfunction and reveals the complex interaction between Pf surgery and chronic L-DOPA treatment. Further investigations are required to better understand the functional state of CM/Pf in BG-related motor and nonmotor disorders, such as Tourette syndrome (Houeto et al., 2005), and to evaluate the efficiency of DBS of this nucleus to manage these pathologies.

\section{References}

Aymerich MS, Barroso-Chinea P, Pérez-Manso M, Muñoz-Patiño AM, Moreno-Igoa M, González-Hernández T, Lanciego JL (2006) Consequences of unilateral nigrostriatal denervation on the thalamostriatal pathway in rats. Eur J Neurosci 23:2099-2108.

Bacci JJ, Kachidian P, Kerkerian-Le Goff L, Salin P (2004) Intralaminar thalamic nuclei lesions: widespread impact on dopamine denervationmediated cellular defects in the rat basal ganglia. J Neuropathol Exp Neurol 63:20-31.

Berardelli A, Rothwell JC, Thompson PD, Hallett M (2001) Pathophysiology of bradykinesia in Parkinson's disease. Brain 124:2131-2146.

Boulet S, Lacombe E, Carcenac C, Feuerstein C, Sgambato-Faure V, Poupard A, Savasta M (2006) Subthalamic stimulation-induced forelimb dyskinesias are linked to an increase in glutamate levels in the substantia nigra pars reticulata. J Neurosci 26:10768-10776.

Caparros-Lefebvre D, Pollak P, Feltin MP, Blond S, Benabid AL. (1999) The effect of thalamic stimulation on levodopa induced dyskinesias. Evaluation of a new target: the center parafascicular median. Rev Neurol (Paris) 155:543-550.

Carli M, Evenden JL, Robbins TW (1985) Depletion of unilateral striatal dopamine impairs initiation of contralateral actions and not sensory attention. Nature 313:679-682.

Castle M, Aymerich MS, Sanchez-Escobar C, Gonzalo N, Obeso JA, Lanciego JL (2005) Thalamic innervation of the direct and indirect basal ganglia pathways in the rat: ipsi- and contralateral projections. J Comp Neurol 483:143-153.

De Groot C (1959) The rat forebrain in stereotaxic coordinate. In: Verhandelingen der koninklijke Nederlandse akademic van wetenschappen. Amsterdam: North-Holland.

Dowd E, Monville C, Torres EM, Dunnett SB (2005) The Corridor Task: a simple test of lateralised response selection sensitive to unilateral dopamine deafferentation and graft-derived dopamine replacement in the striatum. Brain Res Bull 68:24-30.

Fitzsimmons DF, Moloney TC, Dowd E (2006) Further validation of the corridor task for assessing deficit and recovery in the hemi Parkinsonian rat: Restoration of bilateral food retrieval by dopamine receptor agonism. Behav Brain Res 169:352-355.

Freyaldenhoven TE, Ali SF, Schmued LC (1997) Systemic administration of MPTP induces thalamic neuronal degeneration in mice. Brain Res 759:9-17.

Gradinaru V, Mogri M, Thompson KR, Henderson JM, Deisseroth K (2009) Optical deconstruction of parkinsonian neural circuitry. Science 324: 354-359.

Henderson JM, Carpenter K, Cartwright H, Halliday GM. (2000) Loss of thalamic intralaminar nuclei in progressive supranuclear palsy and Parkinson's disease: clinical and therapeutic implications. Brain 123:1410-1421.

Houeto JL, Karachi C, Mallet L, Pillon B, Yelnik J, Mesnage V, Welter ML, Navarro S, Pelissolo A, Damier P, Pidoux B, Dormont D, Cornu P, Agid Y (2005) Tourette's syndrome and deep brain stimulation. J Neurol Neurosurg Psychiatry 76:992-995.

Krauss JK, Pohle T, Weigel R, Burgunder JM (2002) Deep brain stimulation of the centre median-parafascicular complex in patients with movement disorders. J Neurol Neurosurg Psychiatry 72:546-548.

Lacombe E, Khaindrava V, Melon C, Oueslati A, Kerkerian-Le Goff L, Salin P (2009) Different functional basal ganglia subcircuits associated with anti-akinetic and dyskinesiogenic effects of antiparkinsonian therapies. Neurobiol Dis 36:116-125.

Lanciego JL, Rodríguez-Oroz MC, Blesa FJ, Alvarez-Erviti L, Guridi J, Barroso-Chinea P, Smith Y, Obeso JA (2008) Lesion of the centromedian thalamic nucleus in MPTP-treated monkeys. Mov Disord 23:708-715. 
Lundblad M, Andersson M, Winkler C, Kirik D, Wierup N, Cenci MA (2002) Pharmacological validation of behavioural measures of akinesia and dyskinesia in a rat model of Parkinson's disease. Eur J Neurosci 15:120-132.

Marini G, Pianca L, Tredici G (1999) Descending projections araising from the parafascicular nucleus in rats: trajectory of fibers, projection pattern and mapping of terminations. Somatosens Mot Res 16:207-222.

Marshall JF, Richardson JS, Teitelbaum P (1974) Nigrostriatal bundle damage and the lateral hypothalamic syndrome. J Comp Physiol Psychol $87: 808-830$.

Matsumoto N, Minamimoto T, Graybiel AM, Kimura M (2001) Neurons in the thalamic CM-Pf complex supply striatal neurons with information about behaviorally significant sensory events. J Neurophysiol 85:960-976.

Mazzone P, Stocchi F, Galati S, Insola A, Altibrandi MG, Modugno N, Tropepi D, Brusa L, Stefani A (2006) Bilateral implantation of centromedian-parafascicularis complex and GPi: a new combination of unconventional targets for deep brain stimulation in severe Parkinson disease. Neuromodulation 9:221-228.

Minamimoto T, Hori Y, Kimura M (2009) Roles of the thalamic CM-PF complex-Basal ganglia circuit in externally driven rebias of action. Brain Res Bull 78:75-79.

Nanda B, Galvan A, Smith Y, Wichmann T (2009) Effects of stimulation of the centromedian nucleus of the thalamus on the activity of striatal cells in awake rhesus monkeys. Eur J Neurosci 29:588-598.

Orieux G, Francois C, Féger J, Yelnik J, Vila M, Ruberg M, Agid Y, Hirsch EC (2000) Metabolic activity of excitatory parafascicular and pedunculopontine inputs to the subthalamic nucleus in a rat model of Parkinson's disease. Neuroscience 97:79-88.

Oueslati A, Sgambato-Faure V, Melon C, Kachidian P, Gubellini P, Amri M, Kerkerian-Le Goff L, Salin P (2007) High-frequency stimulation of the subthalamic nucleus potentiates L-DOPA-induced neurochemical changes in the striatum in a rat model of Parkinson's disease. J Neurosci 27:2377-2386.

Parr-Brownlie LC, Poloskey SL, Bergstrom DA, Walters JR (2009) Parafascicular thalamic nucleus activity in a rat model of Parkinson's disease. Exp Neurol 217:269-281.

Paxinos G, Watson C. (1986) The rat brain in stereotaxic coordinates. New York: Academic.

Peppe A, Gasbarra A, Stefani A, Chiavalon C, Pierantozzi M, Fermi E, Stanzione
P, Caltagirone C, Mazzone P (2008) Deep brain stimulation of CM/PF of thalamus could be the new elective target for tremor in advanced Parkinson's Disease? Parkinsonism Relat Disord 14:501-504.

Raeva SN (2006) The role of the parafascicular complex (CM-Pf) of the human thalamus in the neuronal mechanisms of selective attention. Neurosci Behav Physiol 36:287-295.

Salin P, Manrique C, Forni C, Kerkerian-Le Goff L (2002) High-frequency stimulation of the subthalamic nucleus selectively reverses dopamine denervation-induced cellular defects in the output structures of the basal ganglia in the rat. J Neurosci 22:5137-5148.

Sedaghat K, Finkelstein DI, Gundlach AL. (2009) Effect of unilateral lesion of the nigrostriatal dopamine pathway on survival and neurochemistry of parafascicular nucleus neurons in the rat-evaluation of time-course and LGR8 expression. Brain Res 1271:83-94.

Sidibé M, Paré JF, Smith Y (2002) Nigral and pallidal inputs to functionally segregated thalamostriatal neurons in the centromedian/parafascicular intralaminar nuclear complex in monkey. J Comp Neurol 447:286-299.

Smith Y, Raju D, Nanda B, Pare JF, Galvan A, Wichmann T (2009) The thalamostriatal systems: anatomical and functional organization in normal and parkinsonian states. Brain Res Bull 78:60-68.

Smith Y, Raju DV, Pare JF, Sidibe M. (2004) The thalamostriatal system: a highly specific network of the basal ganglia circuitry. Trends Neurosci 27:520-527.

Stefani A, Peppe A, Pierantozzi M, Galati S, Moschella V, Stanzione P, Mazzone P (2009) Multi-target strategy for Parkinsonian patients: the role of deep brain stimulation in the centromedian-parafascicularis complex. Brain Res Bull 78:113-118.

Van der Werf YD, Witter MP, Groenewegen HJ (2002) The intralaminar and midline nuclei of the thalamus. Anatomical and functional evidence for participation in processes of arousal and awareness. Brain Res Brain Res Rev 39:107-140.

Wichmann T, Kliem MA, DeLong MR (2001) Antiparkinsonian and behavioral effects of inactivation of the substantia nigra pars reticulate in hemiparkinsonian primates. Exp Neurol 167:410-424.

Winkler C, Kirik D, Björklund A, Cenci MA (2002) L-DOPA-induced dyskinesia in the intrastriatal 6-hydroxydopamine model of Parkinson's disease: relation to motor and cellular parameters of nigrostriatal function. Neurobiol Dis 10:165-186. 